\title{
SZEGÖ KERNEL FUNCTION ON SOME DOMAINS OF INFINITE CONNECTIVITY
}

\author{
By Mitsuru Ozawa
}

1. It is well known that the Szegö kernel function closely relates to a function which maps conformally a given planar $n$-ply connected domain onto the $n$ times covered unit disc ([2], [3], [5], [6]). Recently an interesting eigen-value problem associated with the Szegö kernel function was discussed in [7]. It will be natural to ask for whether these results remain valid or not in the case of the domains of infinite connectivity. Under a somewhat severe condition for the basic domains we shall discuss the nature of the Szegö kernel function and the related function of the domains of infinite connectivity. However it is very plausible to conjecture that the condition and the restriction imposed on in the present paper can be released in a great extent.

We mainly follow a course invented in [3]. In $\S 2$ under a certain condition we discuss an existence theorem which plays a role in the remaining part and seems interesting in itself. In $\S 3$ we shall prove the analyticity of the kernels on the boundary and a boundary relation between them. In $\S 4$ we derive some results using the boundary relation. In order to prove the reproducing property we make a restriction for the function class discussed in $\$ 5$. This restriction chiefly concerns with the Cauchy theorem. $\S 6$ is devoted to derive some estimations for the kernels and then in $\S 7$ we establish a theorem guaranteeing the existence of the discrete eigen-values of a certain integral equation associated with the Szegö kernel function. This theorem is our chief purpose in the present paper. In $\S 8$ we prove that two sorts of kernels introduced in $\S 3$ and in $\S 5$ are coincident with each other. In $\S 9$ we shall introduce a new restriction imposing on the domain, under which we discuss two extremal problems and an exhaustion problem. In $\S 10$ we investigate the behavior of the kernels around a cluster point of the boundary components. Finally in $\S 11$ we offer several problems which seem very important.

2. Let $D$ be a planar infinite domain bounded by an infinite number of circles $C_{\nu}$ defined by an equation $\left|z-a_{\nu}\right|=r_{\nu}, 0<a_{\nu} \leqq 1$, such that $\lim _{\nu \rightarrow \infty} a_{\nu}=0$ and $a_{\nu}$ and $r_{\nu}$ satisfy the condition

$$
\sum_{\substack{j=1 \\ j \neq n}}^{\infty} \frac{r_{j}}{\left|a_{\jmath}-a_{n}\right|^{2}-r_{\jmath}{ }^{2}}<M<\infty,
$$

where $M$ does not depend upon $n$. Then we say that $D$ is of finite type. This nomenclature is justified in $\S 7$. Throughout this paper we assume that $D$ is of finite type. Evidently we have

Received March 20, 1961. 


$$
\pi \sum_{j=1}^{\infty} r_{\jmath} \leqq \pi
$$

Let $B_{\nu}$ be the interior of the circle $C_{\nu}$ and $B$ the sum of all $B_{\nu}$.

LemMa 1. Let $\sigma(z)$ be an integrable function defined on $C=\sum_{\nu=1}^{\infty} C_{\nu}$. There is an integrable function $\Sigma(z)$ on $C$ such that

$$
\int_{C_{\nu}} \Sigma(z) d z=\int_{C_{\nu}} \sigma(z) d z
$$

for each $C_{\nu}, \Sigma(z)$ is regular in $D$ and

for any $w \in B$.

$$
\int_{C} \frac{\Sigma(z)}{z-w} d z=0
$$

Proof. Let $\Sigma(z)$ be of the following form:

$$
\sum_{\nu=1}^{\infty} \frac{\alpha_{\nu}}{2 \pi i\left(z-a_{\nu}\right)}
$$

If $\sum_{\nu=1}^{\infty}\left|\alpha_{\nu}\right|$ is of finite value, then

$$
\sum_{\nu=1}^{\infty} \frac{\alpha_{\nu}}{z-a_{\nu}}
$$

converges absolutely and uniformly in $\bar{D}-U_{\varepsilon}(0)$ to a regular function in $D$, where $U_{\varepsilon}(0)$ is an $\varepsilon$-neighborhood of the origin for any positive number $\varepsilon$. Thus the function is continuous in $\bar{D}-U_{\varepsilon}(0)$ for any $\varepsilon>0$. Next we show that $\Sigma(z)$ is integrable on $C$ if $\sum_{\nu=1}^{\infty}\left|\alpha_{\nu}\right|<\infty$. As a preparation we prove that

$$
I \equiv \int_{0}^{2 \pi} \frac{r_{\nu} d \theta}{\left|z-a_{\mu}\right|} \leqq \frac{2 \pi r_{\nu}}{\sqrt{\left|a_{\nu}-a_{\mu}\right|^{2}-r_{\nu}{ }^{2}}}
$$

for $\left|z-a_{\nu}\right|=r_{\nu} e^{i \theta}$ if $\nu \neq \mu$. If $\nu=\mu$, the left hand side integral is equal to $2 \pi$. By Schwarz's inequality we have

$$
I^{2} \leqq 2 \pi r_{\nu} \int_{0}^{2 \pi} \frac{r_{\nu} d \theta}{\left|z-a_{\mu}\right|^{2}}
$$

Let $w$ be $z-a_{\nu}$, then

$$
\int_{0}^{2 \pi} \frac{d \theta}{\left|z-a_{\mu}\right|^{2}}=\frac{1}{i} \int \frac{d w}{\left(w+a_{\nu}-a_{\mu}\right)\left(\left(a_{\nu}-a_{\mu}\right) w+r_{\nu}{ }^{2}\right)}=2 \pi \frac{1}{\left(a_{\nu}-a_{\mu}\right)^{2}-r_{\nu}{ }^{2}}
$$

by the residue theorem, thus we have

$$
I^{2} \leqq \frac{\left(2 \pi r_{\nu}\right)^{2}}{\left(a_{\nu}-a_{\mu}\right)^{2}-r_{\nu}{ }^{2}} .
$$

By this estimation we have

$$
\begin{aligned}
\int_{C}|\Sigma(z)| d s & \leqq \frac{1}{2 \pi} \int_{C} \sum_{\nu=1}^{\infty} \frac{\left|\alpha_{\nu}\right|}{\left|z-a_{\nu}\right|} d s \\
& =\frac{1}{2 \pi} \sum_{\nu=1}^{\infty}\left[\int_{C_{j}}\left(\sum_{\nu=1}^{j-1}+\sum_{\nu=\jmath+1}^{\infty}\right) \frac{\left|\alpha_{\nu}\right|}{\left|z-a_{\nu}\right|} d s+2 \pi\left|\alpha_{\jmath}\right|\right]
\end{aligned}
$$




$$
\begin{aligned}
& \leqq \frac{1}{2 \pi} \sum_{j=1}^{\infty}\left[\left(\sum_{\nu=1}^{j-1}+\sum_{\nu=j+1}^{\infty}\right)\left|\alpha_{\nu}\right| \int_{C_{j}} \frac{d s}{\left|z-a_{\nu}\right|}\right]+\sum_{j=1}^{\infty}\left|\alpha_{j}\right| \\
& \leqq \frac{1}{2 \pi} \sum_{j=1}^{\infty} \sum_{\nu=1, \neq j}^{\infty}\left|\alpha_{\nu}\right| \frac{2 \pi r_{\jmath}}{\sqrt{\left|a_{\jmath}-a_{\nu}\right|^{2}-r_{\jmath}^{2}}}+\sum_{j=1}^{\infty}\left|\alpha_{j}\right| \\
& =\sum_{\nu=1}^{\infty}\left|\alpha_{\nu}\right| \sum_{j=1, \neq \nu}^{\infty} \frac{r_{\jmath}}{\sqrt{\left|a_{j}-a_{\nu}\right|^{2}-r_{\jmath}^{2}}}+\sum_{j=1}^{\infty}\left|\alpha_{j}\right| \\
& \leqq \sum_{\nu=1}^{\infty}\left|\alpha_{\nu}\right|\left(\sum_{\jmath=1, \neq \nu}^{\infty} \frac{r_{\jmath}}{\left|a_{j}-a_{\nu}\right|^{2}-r_{j}^{2}}\right)^{1 / 2}\left(\sum_{j=1}^{\infty} r_{\jmath}\right)^{1 / 2}+\sum_{j=1}^{\infty}\left|\alpha_{j}\right| \\
& <\left(1+M^{1 / 2} \frac{1}{\sqrt{2}}\right) \sum_{\nu=1}^{\infty}\left|\alpha_{\nu}\right|<\infty,
\end{aligned}
$$

since $D$ is of finite type and $\sum_{\nu=1}^{\infty}\left|\alpha_{\nu}\right|<\infty$. Let $\sigma_{\nu}$ denote the value defined by the integral

$$
\int_{C_{\nu}} \sigma(z) d z
$$

then there holds $\sum_{\nu=1}^{\infty}\left|\sigma_{\nu}\right|<\infty$. Now set $\alpha_{\nu}=\sigma_{\nu}$. Then $\Sigma(z)$ satisfies

$$
\int_{C_{\nu}} \Sigma(z) d z=\frac{1}{2 \pi i} \int_{C_{\nu}} \sum_{\jmath=1}^{\infty} \frac{\sigma_{\jmath}}{z-a_{\jmath}} d z=\sigma_{\nu}
$$

by the uniform convergence of $\Sigma(z)$ on $C_{\nu}$. Let $m_{1}$ be $\min |z-w|$, where the minimum is taken over all $z$ belonging to $\sum_{j=N+1}^{\infty} C_{j}$, and $m_{2}$ be $\min _{z \in C}|z-w|$ for a given $w \in B$. Then we have

$$
\begin{aligned}
& \left|\sum_{j=N+1}^{\infty} \int_{C_{j}} \frac{1}{z-w} \sum_{\nu=1}^{\infty} \frac{\sigma_{\nu}}{2 \pi i\left(z-a_{\nu}\right)} d z\right| \\
& \leqq \frac{1}{2 \pi m_{1}} \sum_{j=N+1}^{\infty} \int_{C_{j}} \sum_{\nu=1}^{\infty} \frac{\left|\sigma_{\nu}\right|}{\left|z-a_{\nu}\right|} d s<\varepsilon
\end{aligned}
$$

by the integrability of $\Sigma(z)$, and further we have

$$
\begin{aligned}
& \left|\sum_{j=1}^{\infty} \int_{C_{j}} \frac{1}{z-w} \sum_{\nu=N+1}^{\infty} \frac{\sigma_{\nu}}{2 \pi i\left(z-a_{\nu}\right)} d z\right| \\
\leqq & \sum_{j=1}^{\infty} \sum_{\nu=N+1}^{\infty} \frac{\left|\sigma_{\nu}\right|}{2 \pi} \int_{C_{j}} \frac{d s}{|z-w|\left|z-a_{\nu}\right|} \\
\leqq & \frac{1}{2 \pi m_{2}} \sum_{j=1}^{\infty} \sum_{\nu=N+1}^{\infty}\left|\sigma_{\nu}\right| \int_{C_{j}} \frac{d s}{\left|z-a_{\nu}\right|} \\
\leqq & \frac{1}{2 \pi m_{2}} \sum_{\nu=N+1}^{\infty}\left|\sigma_{\nu}\right|\left(\sum_{j=1, \neq \nu}^{\infty} \frac{r_{j}}{\sqrt{\left|a_{j}-a_{\nu}\right|^{2}-r_{j}^{2}}}+2 \pi\right) \\
\leqq & \frac{M}{2 \pi m_{2}} \sum_{\nu=N+1}^{\infty}\left|\sigma_{\nu}\right|<\varepsilon .
\end{aligned}
$$

These show that

$$
\left|\int_{C} \Sigma(z) \frac{d z}{z-w}\right| \leqq\left|\sum_{\nu=1}^{N} \sum_{\nu=1}^{N} \int_{C_{j}} \frac{\sigma_{\nu}}{2 \pi i(z-w)\left(z-a_{\nu}\right)} d z\right|+2 \varepsilon .
$$

The first term in the right hand side is equal to zero by the residue theorem, 
which leads to the desired result, that is,

$$
\int_{C} \Sigma(z) \frac{d z}{z-w}=0
$$

for any $w \in B$.

3. Let $L^{2}$ be a class of all complex-valued functions $\mu(s)$ defined on $C$ which have a finite Lebesgue integral

$$
\|\mu\|^{2}=\int_{C}|\mu(s)|^{2} d s .
$$

This class $L^{2}$ is a Hilbert space with the scalar product

$$
(\mu, \nu)=\int_{C} \mu(s) \overline{\nu(s)} d s
$$

defined for any pair of elements $\mu(s)$ and $\nu(s)$ in $L^{2}$. We consider the linear subspace $\Lambda^{2}$ of $L^{2}$ consisting of these functions $\mu(s) \in L^{2}$ which satisfy the identity

$$
\int_{C} \frac{\mu(s)}{z-w} d z=0, \quad z=z(s), \quad w \in B
$$

It is clear that $\Lambda^{2}$ is closed in $L^{2}$. Then, for any element $M(s) \in L^{2}$, we can select a function $\mu(s)$ in $\Lambda^{2}$ which is nearest to $M(s)$ in the sense that

$$
\|M(s)-\mu(s)\|^{2}=\int_{C}|M(s)-\mu(s)|^{2} d s=\text { minimum. }
$$

Then we have the decomposition

$$
M(s)=\mu(s)+\overline{\nu(s) z^{\prime}(s)}, \quad \mu(s), \nu(s) \in \Lambda^{2},
$$

which is unique, and an orthogonality relation

for any $\nu_{1} \in \Lambda^{2}$.

$$
\left(M-\mu, \nu_{1}\right)=0
$$

In the above decomposition we choose for $M(s)$ the particular function

$$
M(s)=\frac{1}{2 \pi i} \frac{1}{z(s)-t},
$$

where $t$ is a fixed point in $D$, and we have

$$
\frac{1}{2 \pi i} \frac{1}{z(s)-t}=\rho(z, t)+\overline{k(z, t) z^{\prime}(s)}, \quad \rho(z(s), t), k(z(s), t) \in A^{2} .
$$

We can set up the pair of analytic functions

$$
k(w, t)=\frac{1}{2 \pi i} \int_{C} \frac{k(z, t)}{z-w} d z, \quad \rho(w, t)=\frac{1}{2 \pi i} \int_{C} \frac{\rho(z, t)}{z-w} d z,
$$

and we set

$$
l(w, t)=\frac{1}{2 \pi i} \frac{1}{w-t}-\rho(w, t) .
$$


LEMma 2. Let $\sigma(z)$ be any integrable function defined on $C$ with

$$
\int_{C}|\sigma(z)| d s<\infty
$$

which satisfies the relation

$$
\int_{C} \frac{\sigma(z)}{z-w} d z=0, \quad w \in B
$$

The indefinite integral $F(w)$ of the analytic function

$$
f(w)=\frac{1}{2 \pi i} \int_{C} \frac{\sigma(z)}{z-w} d z
$$

in $D$ is continuous in $D+C-U_{\varepsilon}(0)$ or more precisely in the neighborhood of each $C_{\nu}$ in $D$ including $C_{\nu}$ and its boundary value is equal to

$$
\int^{z} \sigma(z) d z
$$

up to an additive constant.

To show this we remark in the first place that we may suppose

$$
\int_{C_{\nu}} \sigma(z) d z=0
$$

for each $C_{\nu}$. Indeed, there exists a regular function $\Sigma(z)$ satisfying the conditions

and

$$
\int_{C_{\nu}} \Sigma(z) d z=\int_{C_{\nu}} \sigma(z) d z
$$

$$
\int_{C} \frac{\Sigma(z)}{z-w} d z=0
$$

by Lemma 1, and hence $\sigma(z)-\Sigma(z)$ satisfies the conditions required. On the other hand the indefinite integral of the analytic function

$$
\frac{1}{2 \pi i} \int_{C} \frac{\Sigma(z)}{z-w} d z, \quad w \in D
$$

is continuous in the neighborhood of each $C_{\nu}$ in $D$ including $C_{\nu}$ and its boundary value is equal to

$$
\int^{z} \Sigma(z) d z
$$

up to an additive constant, since $\Sigma(z)$, by its construction, is an absolutely and uniformly convergent series in the neighborhood of $C_{\nu}$ in $D$ including $C_{\nu}$ and hence $\Sigma(z)$ is regular on $C_{\nu}$. Therefore we may suppose, with no loss of generality, that there holds

$$
\int_{C_{\nu}} \sigma(z) d z=0
$$

for each $C_{\nu}$. Now we have finished all the necessary preparations which enable 
us to prove Lemma 2, and the remaining part of the proof is a course due to [3] ad verbum. And finally we have our Lemma 2.

Let $m$ be the minimum distance from $w \in D$ to $C$, then we have

and

$$
\begin{aligned}
|k(w, t)|^{2} & \leqq \frac{\|k(z, t)\|^{2}}{4 \pi^{2}} \int_{C} \frac{d s}{|z-w|^{2}} \\
& =\frac{\|k(z, t)\|^{2}}{2 \pi} \sum_{\jmath=1}^{\infty} \frac{r_{\jmath}}{\left|a_{\jmath}-w\right|^{2}-r_{\jmath}^{2}} \\
& \leqq \frac{\|k(z, t)\|^{2}}{2 \pi m} \sum_{\jmath=1}^{\infty} \frac{r_{\jmath}}{\left|a_{\jmath}-w\right|+r_{\jmath}}
\end{aligned}
$$

$$
\begin{aligned}
\sum_{\jmath=1}^{\infty} \frac{r_{\jmath}}{\left|w-a_{\jmath}\right|+r_{\jmath}} & \leqq \sum_{\jmath=1}^{\nu-1} \frac{r_{\jmath}}{\left|a_{\jmath}-a_{\nu}-r_{\nu}\right|+r_{\jmath}}+\frac{1}{2}+\sum_{\jmath=\nu+1}^{\infty} \frac{r_{\jmath}}{\left|a_{\jmath}-a_{\nu}+r_{\nu}\right|+r_{\jmath}} \\
& \leqq \frac{1}{\left|a_{\nu-1}-a_{\nu}-r_{\nu}\right|} \sum_{\jmath=1}^{\nu-1} r_{\jmath}+\frac{1}{2}+\frac{1}{\left|a_{\nu}-r_{\nu}-a_{\nu+1}\right|} \sum_{j=\nu+1}^{\infty} r_{\jmath}<\infty .
\end{aligned}
$$

This shows that $|k(w, t)|=O\left(m^{-1 / 2}\right)$. Similarly we have $|\rho(w, t)|=O\left(m^{-1 / 2}\right)$, $|l(w, t)|=O\left(m^{-1 / 2}\right)$, when $w$ is bounded away from $t$ in the last estimation. A similar discussion as in [3] leads to an important fact that the functions $k(z, t)$ and $l(z, t)$ defined by the Cauchy integral throughout $D$ are analytic in $D$ and on each $C_{\nu}$, except for the simple pole of $l(z, t)$ at $z=t$ and they satisfy the boundary relation

$$
l(z, t)=\overline{k(z, t) z^{\prime}(s)}
$$

everywhere on each $C_{\nu}$. This is the one playing the most important role in the theory of the Szegö kernel function.

4. In $\S 3$ we have shown an orthogonal relation $\left(M-\mu, \nu_{1}\right)=0$ for any $\nu_{1} \in \Lambda^{2}$. Therefore we have

$$
\int_{C} \overline{l(z, t)} \rho(z, w) d s=0 .
$$

By this relation we have

$$
\begin{aligned}
0 & =\int_{C} \overline{\rho(z, w)} l(z, t) d s=\int_{C} \overline{\rho(z, w)}\left(\frac{1}{2 \pi i} \frac{1}{z-t}-\rho(z, t)\right) d s \\
& =\frac{1}{2 \pi i} \int_{C} \frac{\overline{\rho(z, w)}}{z-t} d s-\int_{C} \overline{\rho(z, w)} \rho(z, t) d s
\end{aligned}
$$

and

$$
\begin{aligned}
k(t, w) & =\frac{1}{2 \pi i} \int_{C} \frac{k(z, w)}{z-t} d z=\frac{1}{2 \pi i} \int_{C} \frac{\overline{l(z, w)}}{z-t} d s \\
& =\frac{1}{4 \pi^{2}} \int_{C} \frac{d s}{(z-t)(\bar{z}-\bar{w})}-\frac{1}{2 \pi i} \int_{C} \frac{\overline{\rho(z, w)}}{z-t} d s
\end{aligned}
$$

which show that there holds an important relation 
where we set

$$
\rho_{2}(t, w)+k(t, w)=\Gamma(t, w)
$$

$$
\rho_{2}(t, w)=\int_{C} \overline{\rho(z, w)} \rho(z, t) d s
$$

and

$$
\Gamma(t, w)=\frac{1}{4 \pi^{2}} \int_{C} \frac{d s}{(z-t)(\bar{z}-\bar{w})} .
$$

Evidently we have the Hermitian character of $\Gamma$ and $\rho_{2}$, that is, $\Gamma(t, w)$ $=\overline{\Gamma(w, t)}$ and $\rho_{2}(t, w)=\overline{\rho_{2}(w, t)}$. By its definition $\rho_{2}(t, w)$ is positive semi-definite, that is, for any $\varphi \in L^{2}$

$$
\int_{C} \int_{C} \rho_{2}(t, w) \overline{\varphi(t)} \varphi(w) d s_{t} d s_{w}=\left.\int_{C} \int||_{C} \rho(t, z) \overline{\varphi(t)} d s_{t}\right|^{2} d s_{z} \geqq 0 .
$$

On the other hand there holds

$$
\begin{aligned}
(k(z, w), k(z, t)) & =\int_{C} l(z, t) \overline{l(z, w)} d s \\
& =\Gamma(t, w)-\frac{1}{2 \pi i} \int_{C} \frac{\overline{\rho(z, w)}}{z-t} d s-\frac{1}{2 \pi i} \int_{C} \frac{\overline{\rho(z, t)}}{z-w} d s+\rho_{2}(t, w) \\
& =\Gamma(t, w)-\rho_{2}(t, w)=k(t, w),
\end{aligned}
$$

in particular,

$$
k(t, t)=\|k(z, t)\|^{2}=\min _{\mu \in \Lambda^{2}}\left\|\frac{1}{2 \pi i(z-t)}-\mu\right\|^{2} .
$$

In the above proof we do not use the reproducing property, which is not yet settled.

5. The reproducing property of the Szegö kernel function is an immediate consequence of the Cauchy theorem in the classical case of any finitely connected analytic domain. However we cannot, without any verification, conclude that the Cauchy theorem remains valid in our case. Therefore we need to replace $\Gamma^{2}$ instead of $\Lambda^{2}$. Let $\Phi$ be a class of functions regular in $D$ and having the $L^{2}$ integrable boundary value $\psi(z(s)$ ) almost everywhere on $C$ and satisfying the Cauchy integrable formula

$$
\varphi(t)=\frac{1}{2 \pi i} \int_{C} \frac{\varphi(z)}{z-t} d z, \quad t \in D .
$$

Let $\Gamma^{2}$ be a class of $L^{2}$ function $\mu(z)$ on $C$ such that the condition

$$
\int_{C} \mu(z) \varphi(z) d z=0
$$

holds for any $\varphi \in \Phi$.

For any $L^{1}$ integrable function $\psi(z)$ on $C$, the function defined by 


$$
\varphi(t)=\frac{1}{2 \pi i} \int_{C} \frac{\psi(z)}{z-t} d z
$$

has the jump $\psi(z)$ almost everywhere on $C$ when either the inner normal boundary value or the outer one exists. This is a well known theorem due to Plemelj.

Thus in our case there remains valid

$$
\int_{C} \frac{\phi(z)}{z-t} d z=0, \quad t \in B
$$

whence follows a fact that $1 /(z-t), t \in B$, belongs to the class $\Gamma^{2}$. Since $1 /(z-t), t \in B$, also belongs to the class $\Phi$, any function $\mu(s) \in \Gamma^{2}$ satisfies

$$
\int_{C} \mu(z) \frac{d z}{z-t}=0, \quad t \in B,
$$

which shows that $\Gamma^{2} \subset \Lambda^{2}$. The class $\Gamma^{2}$ is also evidently closed in $L^{2}$. This leads again to the following decomposition:

$$
\frac{1}{2 \pi i(z-t)}=\rho(z, t)+\overline{k(z, t) z^{\prime}}, \quad t \in D, \rho(z, t) \in \Gamma^{2} .
$$

And further we can see that the orthogonality relation $\left(k(z, t) z^{\prime}, \nu(z)\right)=0$ holds for any $\nu \in \Gamma^{2}$ and hence that

$$
\int_{C} \frac{k(z, t)}{z-w} d z=0, \quad w \in B
$$

This implies that $k(z, t) \in \Lambda^{2}$. Hence we can apply the same reasoning as in $\S 3$ and we have the existence of the kernels $k(z, t), \rho(z, t)$ and $l(z, t)$ which are analytic on each $C_{\nu}$ and satisfy the boundary relation

$$
l(z(s), t)=\overline{k(z(s), t) z^{\prime}(s)} .
$$

Then we have the reproducing property of $k(z, t)$ for any $\varphi \in \Phi$ :

$$
\begin{aligned}
(\varphi(z), k(z, t)) & =\int_{C} \varphi(z) l(z, t) d z \\
& =\frac{1}{2 \pi i} \int_{C} \frac{\varphi(z)}{z-t} d z-\int_{C} \varphi(z) \rho(z, t) d z=\varphi(t) .
\end{aligned}
$$

And further we have $k(z, t) \in \Phi$ and $(k(z, w), k(z, t))=k(t, w)$. The reproducing property is a key property of the kernel and formally we can say that there holds an extremal property

$$
\frac{1}{k(t, t)}=\left\|\frac{k(z, t)}{k(t, t)}\right\|^{2} \leqq\left\|\frac{\varphi(z)}{\varphi(t)}\right\|^{2}
$$

for any $L^{2}$ analytic function $\varphi$ in $D$ belonging to the class $\Phi$. This leads to the monotoneity of the kernel, that is, if $D_{1} \subset D_{2}$, then

$$
k_{D_{1}}(t, t) \geqq k_{D_{2}}(t, t) \text {. }
$$


Antisymmetricity of $\rho(z, t)$ is now easily concluded as follows:

$$
\begin{aligned}
0= & \int_{C} l(z, t) k(z, w) d s \\
= & \frac{1}{(2 \pi i)^{2}} \int_{C} \frac{1}{(z-t)(z-w)} d z-\frac{1}{2 \pi i} \int_{C} \frac{\rho(z, w)}{z-t} d z \\
& -\frac{1}{2 \pi i} \int_{C} \frac{\rho(z, t)}{z-w} d z+\int_{C} \rho(z, t) \rho(z, w) d z=-\rho(t, w)-\rho(w, t) .
\end{aligned}
$$

It should be here remarked that the kernel $k(z, t)$ has not the reproducing property for any $L^{2}$ analytic function in $D$. This will be proved in $\S 11$.

6. Let $\Gamma_{X}(z, \zeta)$ and $k_{X}(z, \zeta)$ be the corresponding quantities of $\Gamma$ and $k$ in the domain $X$, respectively. Then we have

$$
k_{\rho<|z|<1}(z, \zeta)=\frac{1}{2 \pi} \sum_{\nu=0}^{\infty} \frac{1}{1+\rho^{2 \nu+1}}\left[z^{\nu \bar{\rho}_{\nu}}+\rho^{2 \nu+1} z^{-\nu-1} \bar{\zeta}^{-\nu-1}\right]
$$

and

$$
k_{\rho<|z|}(z, \zeta)=\Gamma_{\rho<|z|}(z, \zeta)=\frac{1}{2 \pi} \sum_{\nu=0}^{\infty} \frac{\rho^{2 \nu+1}}{(z \bar{\zeta})^{\nu+1}} .
$$

Thus we have

$$
\Gamma_{\rho<|z|}(z, z)-k_{\rho<|z|<1}(z, z)=\frac{1}{2 \pi} \sum_{\nu=0}^{\infty} \frac{\rho^{2 \nu+1}}{|z|^{2 \nu+2}} \frac{\rho^{2 \nu+1}}{1+\rho^{2 \nu+1}}-\frac{1}{2 \pi} \sum_{\nu=0}^{\infty} \frac{|z|^{2 \nu}}{1+\rho^{2 \nu+1}},
$$

and hence we see

$$
\lim _{|z| \rightarrow \rho}\left(\Gamma_{\rho<|z|}(z, z)-k_{\rho<|z|<1}(z, z)\right)=0 .
$$

Let $D_{j}$ and $R_{\jmath}$ be the domains defined by $\left|z-a_{\jmath}\right|>r_{\jmath}$ and $r_{\jmath}<\left|z-a_{\jmath}\right|<P_{\jmath}$, respectively, where $P_{\jmath}$ is determined in such a manner that $R_{\jmath} \subset D$ and $R_{\jmath}$ is maximal. Then by the monotoneity we have

$$
\sum_{\jmath=1, \neq \nu}^{\infty} k_{D_{j}}(z, z)+k_{D_{\nu}}(z, z)-k_{R_{\nu}}(z, z) \leqq \Gamma_{D}(z, z)-k_{D}(z, z) \leqq \sum_{j=1, \neq \nu}^{\infty} k_{D_{j}}(z, z)
$$

in $R_{\nu}$, since there holds

$$
\Gamma_{D}(z, z)=\sum_{j=1}^{\infty} \Gamma_{D_{j}}(z, z)=\sum_{j=1}^{\infty} k_{D_{j}}(z, z)
$$

Let $z$ tend to a point $\zeta$ on $C_{\nu}$, then we have

$$
\lim _{z \rightarrow \zeta}\left(\Gamma_{D}(z, z)-k_{D}(z, z)\right)=\lim _{z \rightarrow \zeta} \sum_{j=1, \neq \nu}^{\infty} k_{D_{j}}(z, z) .
$$

On the other hand we have

$$
k_{D_{j}}(z, z)=\frac{1}{2 \pi} \frac{r_{\jmath}}{\left|z-a_{\jmath}\right|^{2}-r_{\jmath}^{2}},
$$

and therefore we can conclude that

$$
\lim _{z \rightarrow \zeta}\left(\Gamma_{D}(z, z)-k_{D}(z, z)\right)=\frac{1}{2 \pi} \sum_{j=1, \neq \nu}^{\infty} \frac{r_{\jmath}}{\left|\zeta-a_{\jmath}\right|^{2}-r_{\jmath}^{2}}, \quad \zeta \in C_{\nu} .
$$


The right hand side series is uniformly and absolutely convergent by our condition (A), and hence it is also uniformly bounded on $C$.

7. Now we shall consider an integral equation

$$
f(z)=\lambda^{2} \int_{C} \rho_{2}(z, t) f(t) d s_{t}
$$

for $f$ being $L^{2}$ analytic in $D$. We have already shown that $\Gamma(z, z)-k(z, z)$ $=\rho_{2}(z, z)$ and its uniform boundedness holds on $C$, therefore

This shows that

$$
\begin{aligned}
\left|\rho_{2}(z, t)\right| & \leqq \int_{C}|\rho(w, t)||\rho(w, z)| d s_{w} \\
& \leqq\left(\int_{C}|\rho(w, t)|^{2} d s\right)^{1 / 2}\left(\int_{C}|\rho(w, z)|^{2} d s\right)^{1 / 2} \\
& =\rho_{2}(t, t)^{1 / 2} \rho_{2}(z, z)^{1 / 2} .
\end{aligned}
$$

$$
\begin{aligned}
\int_{C} \int_{C}\left|\rho_{2}(z, t)\right|^{2} d s_{z} d s_{t} & \leqq\left(\int_{C} \rho_{2}(z, z) d s\right)^{2} \\
& \leqq M^{2}\left(\sum_{\jmath=1}^{\infty} 2 \pi r_{\jmath}\right)^{2}<\infty
\end{aligned}
$$

Thus we have the following theorem.

Theorem 1. Let $D$ be a domain of finite type. Then Hilbert-Schmidt theory is applicable to our integral equation

$$
f(z)=\lambda^{2} \int_{C} \rho_{2}(z, t) f(t) d s
$$

and therefore there exists a finite number of eigen-values of finite value and a finite number of the associating eigenfunctions. However there may exist an infinite number of eigenfunctions belonging to the eigen-value $\lambda=\infty$. Further, the eigenfunctions $\phi_{\nu}$ and $\phi_{\mu}$ belonging to the different eigen-values $\lambda_{\nu}{ }^{2}$ and $\lambda_{\mu}{ }^{2}$ are orthogonal and the eigenfunction $\phi_{\nu}{ }^{(\rho)}(z)(\rho=1,2, \cdots, p)$ which belong to an eigen-value $\lambda_{\nu}{ }^{2}$ of degeneracy $p-1$ may be supposed orthogonal.

This theorem shows that the situation is the same as in the case of finitely connected domain as far as we concern with our eigen-value problem. The word "of finite type" is due to this fact. Singh discussed the problem recently in a finitely connected analytic case [7]. Since several results can be obtained ad verbum as in [7], we do not enter into a further discussion in this tendency.

Now we shall mention here some examples.

Example 1. Let $a_{n}=1 / n$ and $r_{n}=1 / n^{4}, n=2,3, \cdots$.

Then $D$ is of finite type.

Example 2. Let $a_{n}=1 / n$ and $r_{n}=1 / 10^{n}, n=1,2, \cdots$.

Then $D$ is of finite type. 
Example 3. Let $a_{n}=1 / n$ and $r_{n}=1 / 2 n(n+1), n=1,2, \cdots$.

Then $D$ is not of finite type.

8. In $\S 3$ we explained the decomposition

$$
\frac{1}{2 \pi i(z-t)}=\rho(z, t)+\overline{k(z, t) z^{\prime}}, \quad t \in D, \quad \rho, k \in A^{2} .
$$

In $\S 5$ we also explained the decomposition

$$
\frac{1}{2 \pi i(z-t)}=\rho(z, t)+\overline{k(z, t) z^{\prime}}, \quad t \in D, \rho \in \Gamma^{2}, k \in \Lambda^{2} .
$$

In order to avoid the eventual misunderstanding we shall denote the former decomposition in the following form:

$$
\frac{1}{2 \pi i(z-t)}=P(z, t)+\overline{K(z, t) z^{\prime}} .
$$

In $\S \S 3$ and 5 we proved the inclusions $\Gamma^{2} \subset \Lambda^{2} \subset L^{2}$ and $\Phi \subset \Lambda^{2}$. And further we proved

$$
\frac{1}{z-w} \in \Gamma^{2} \cap \Phi \subset \Lambda^{2}, \quad w \in B,
$$

and

$$
k(z, t) \in \Phi \subset \Lambda^{2} .
$$

By the theorem of Plemelj we can see that $P(z, t), \rho(z, t)$ and $K(z, t)$ belong to the class $\Phi$. By these preparations we next prove that $k(z, t)=K(z, t)$ and $\rho(z, t)=P(z, t)$.

Indeed, since $L(z, w)=1 / 2 \pi i(z-w)-P(z, w)$ is orthogonal to the class $\Lambda^{2}$, there holds

$$
\begin{aligned}
0 & =\int_{C} \overline{k(z, t)} L(z, w) d s \\
& =\int_{C} \overline{k(z, t)}\left(\frac{1}{2 \pi i(z-w)}-P(z, w)\right) d s
\end{aligned}
$$

which shows that

$$
P(t, w)=\int_{C} \overline{k(z, t)} P(z, w) d s=\frac{1}{2 \pi i} \int_{C} \frac{\overline{k(z, t)}}{z-w} d s .
$$

On the other hand we have

$$
\begin{aligned}
& \frac{1}{2 \pi i} \int_{C} \frac{k(z, t)}{z-w} d s-\rho(t, w) \\
= & \int_{C} \overline{k(z, t)} l(z, w) d s=\int_{C} l(z, t) l(z, w) d z \\
= & \frac{-1}{4 \pi^{2}} \int_{C} \frac{d z}{(z-t)(z-w)}-\frac{1}{2 \pi i} \int_{C} \frac{\rho(z, t)}{z-w} d z
\end{aligned}
$$




$$
-\frac{1}{2 \pi i} \int_{C} \frac{\rho(z, w)}{z-t} d z+\int_{C} \rho(z, t) \rho(z, w) d z=0 .
$$

Therefore we see that $\rho(t, w) \equiv P(t, w)$. Thus $k(z(s), t) \equiv K(z(s), t), z(s) \in C$, remains valid, which leads to the desired result $k(z, t) \equiv K(z, t)$.

9. We now introduce a new restriction imposed on the domain $D$. We say, in general, that $D$ is of infinite island type if there exists a closed subdomain $D^{\prime}$ of $D+\{0\}$ such that the complementary set consists of an infinite number of disc islands and $\Gamma(z, w)$ is uniformly bounded in $D^{\prime} \times D^{\prime}$ and $\partial D^{\prime}$ contains the origin. There are many domains of infinite island type. Example 2 in $\S 7$ is surely of this type. Indeed, we see that

$$
\Gamma_{D}(z, w)=\frac{1}{2 \pi} \sum_{n=1}^{\infty} \frac{r_{n}}{\left(z-a_{n}\right)\left(\bar{w}-a_{n}\right)-r_{n}{ }^{2}}
$$

is uniformly bounded for $(z, w) \in D_{e} \times D_{e}$, where $D_{e}$ is a closed subdomain of $D+\{0\}$ defined by $\cap_{n=1}^{\infty}\left\{z ;\left|z-a_{n}\right| \geqq 1 / e^{n}\right\}$. In this case we have in $D_{e} \times D_{e}$

$$
\left|\Gamma_{D}(z, w)\right| \leqq \frac{1}{2 \pi} \sum_{n=1}^{\infty} \frac{10^{-n}}{e^{-2 n}-10^{-2 n}}<\infty .
$$

In general, since $k_{D}(z, z) \leqq \Gamma_{D}(z, z)$ and $\left|k_{D}(z, w)\right|^{2} \leqq k_{D}(z, z) \cdot k_{D}(z, w), k_{D}(z, z)$, $k_{D}(z, w)$ are uniformly bounded in $D^{\prime}, D^{\prime} \times D^{\prime}$, respectively. Since, by Schwarz's inequality, we have

$$
\begin{aligned}
\left|\rho_{D}(z, w)\right|^{2} & \leqq \int_{C}\left|k_{D}(t, z)\right|^{2} d s \int_{C}\left|\rho_{D}(t, w)\right|^{2} d s \\
& =k_{D}(z, z) \cdot \rho_{2 D}(w, w)=k_{D}(z, z)\left(\Gamma_{D}(w, w)-k_{D}(w, w)\right),
\end{aligned}
$$

we can see that $\rho_{D}(z, w)$ is also uniformly bounded in $D^{\prime} \times D^{\prime}$. Hence $l_{D}(z, w)$ is also so, when $z$ is bounded away from $w$. By its expression it is evident that $\Gamma_{D}(z, w)=\Gamma_{D}(\bar{w}, \bar{z})$ and $\Gamma_{D}(z, z)=\Gamma_{D}(\bar{z}, \bar{z})$, and on the other hand there holds an inequality $\left|\Gamma_{D}(z, w)\right|^{2} \leqq \Gamma_{D}(z, z) \Gamma_{D}(w, w)$. If $D$ is of infinite island type, then we can select $D^{\prime}$ as a symmetric domain with respect to the real axis and hence we can choose an infinite number of circles $\gamma_{n}$ each of which lies in $D^{\prime}$ and runs through the origin and separates the circles $C_{n}$ and $C_{n+1}$ and has its centre on the real axis. The length of the periphery of $\gamma_{n}$ tends to zero as $n$ tends to the infinity.

Let $\left\{D_{n}\right\}$ be an exhaustion of $D$ in a certain sense and $\mathfrak{F}_{n}$ be a class of functions, in which an extremal problem is considered. Under what condition for $D$ and for the problem does the extremal function of the problem tend to an extremal function of the corresponding problem on $D$ ? Is the extremal function for $D$ unique? This problem, which we say the exhaustion problem, is one of the most important problems to be considered, and there are numerous papers for the various extremal problems.

Let $\mathfrak{B}_{X}$ be a class of bounded single-valued regular function in $X$ such that 
$|f(z)| \leqq 1, z \in D$. Then $\mathfrak{B}_{D}$ is a subclass of $\Phi$. Indeed, we have for $w \in D$

$$
\frac{1}{2 \pi i} \int_{C} \frac{f(z)}{z-w} d z=\frac{1}{2 \pi i} \int_{\sum_{\jmath=1}^{N} C_{j+\gamma_{N}}} \frac{f(z)}{z-w} d z+\frac{1}{2 \pi i} \int_{\sum_{\jmath=N+1}^{\infty} C_{j-\gamma}-\gamma_{N}} \frac{f(z)}{z-w} d z,
$$

where $\gamma_{N}$ has already been defined. The first term of the right hand side is equal to $f(w)$ by the residue theorem and the second one has its modulus less than $\varepsilon$. Therefore we have

$$
f(w)=\frac{1}{2 \pi i} \int_{C} \frac{f(z)}{z-w} d z, \quad w \in D,
$$

which shows that $\mathfrak{B}_{D} \subset \Phi$. In the above discussion we have not made use of a fact that $D$ is of infinite island type.

Since it is not yet settled whether there is the multiplication in $\Phi$ or not and whether $k(z, w) / l(z, w)$ is bounded or not, we need to make a detour and to restrict ourselves to the domain of infinite island type.

Let $D$ be of infinite island type. In this case we have for $f(z) \in \mathfrak{B}_{D}$

$$
f^{\prime}(z)=2 \pi i \int_{C} l_{D}(t, z)^{2} f(t) d t
$$

In fact, we have for $z \in D^{\prime}-\bar{U}_{\varepsilon}(0)$

$$
\int_{\Sigma_{j=1}^{N} C_{j+\gamma_{N}}} \frac{f(t)}{t-z} \rho_{D}(t, z) d t=2 \pi i f(z) \cdot \rho_{D}(z, z)=0,
$$

since $\rho_{D}(z, z)=0$. And further we have

$$
\left|\int_{\sum_{j=N+1}^{\infty} C_{j}-\gamma_{N}} \frac{f(t)}{t-z} \rho_{D}(t, z) d t\right|<\varepsilon,
$$

since

$$
\left|\int_{\Sigma_{N+1}^{\infty} C_{j}} \frac{f(t)}{t-z} \rho_{D}(t, z) d t\right| \leqq \int_{\Sigma_{N+1}^{\infty} C_{j}} \frac{\left|\rho_{D}(t, z)\right|}{|t-z|} d s<\frac{\varepsilon}{2}
$$

and

$$
\left|\int_{\gamma_{N}} \frac{f(t)}{t-z} \rho_{D}(t, z) d t\right| \leqq M \int_{r_{N}} d s<\frac{\varepsilon}{2} .
$$

By these estimations we have

$$
\int_{C} \frac{f(t)}{t-z} \rho_{D}(t, z) d t=0 .
$$

Quite similarly we can see that

$$
\int_{C} \rho_{D}(t, z)^{2} f(t) d t=0, \quad f \in \mathfrak{B}_{D}, \quad z \in D^{\prime}-\bar{U}_{\varepsilon}(0) .
$$

Therefore we can conclude that

$$
f^{\prime}(z)=2 \pi i \int_{C} l_{D}(t, z)^{2} f(t) d t
$$


for any $f(t) \in \mathfrak{B}_{D}$ and for any $z \in D^{\prime}-\bar{U}_{\varepsilon}(0)$. The analyticity of each side implies that this identity holds for any $f(t) \in \mathfrak{B}_{D}$ and for any $z \in D$.

By the identity we have

$$
\left|f^{\prime}(z)\right| \leqq 2 \pi \int_{C}\left|l_{D}(t, z)\right|^{2} d s=2 \pi \int_{C} \overline{k_{D}(t, z)} k_{D}(t, z) d s=2 \pi k_{D}(z, z),
$$

which shows that

$$
\sup _{\mathfrak{B}_{D}}\left|f^{\prime}(z)\right| \leqq 2 \pi k_{D}(z, z) .
$$

On the other hand it is well known that

$$
\sup _{\mathfrak{B}_{D(n)}}\left|g^{\prime}(z)\right|=2 \pi k_{D(n)}(z, z) \geqq 2 \pi k_{D}(z, z),
$$

where $D(n)$ is the subdomain of $D$ bounded by the circles $C_{\nu}, \nu=1, \cdots, n$, and a circle $\gamma_{n}([2],[3],[5],[6])$. This and the compactness of the class $\mathfrak{B}_{D}$ and the monotoneity of the functional in the sense of Ahlfors-Beurling [1] imply that

$$
\sup _{\mathfrak{B}_{D}}\left|f^{\prime}(z)\right|=2 \pi \lim _{n \rightarrow \infty} k_{D(n)}(z, z)=2 \pi k_{D}(z, z),
$$

taking a suitable subsequence, if necessary.

For any $n \geqq N+1$, we have

$$
\int_{r_{n}}\left|k_{D}(t, w)\right|^{2} d s<\varepsilon, \quad w \in D^{\prime},
$$

and further we have

$$
\int_{\Sigma_{n}^{\infty} C_{j}}\left|k_{D}(t, w)\right|^{2} d s<\varepsilon, \quad w \in D
$$

since $k_{D} \in L^{2}$. Then we see that

$$
\begin{aligned}
& \int_{\sum_{1}^{n} C j+\gamma n}\left|k_{D}(t, z)-k_{D(n)}(t, z)\right|^{2} d s \\
= & k_{D(n)}(z, z)-k_{D}(z, z)-k_{D}(z, z)+\int_{\Sigma_{1}^{n} C j+\gamma_{n}}\left|k_{D}(t, z)\right|^{2} d s \\
\leqq & k_{D(n)}(z, z)-k_{D}(z, z)+\int_{\sum_{n+1}^{\infty} C_{j+\gamma_{n}}}\left|k_{D}(t, z)\right|^{2} d s<3 \varepsilon, \quad z \in D^{\prime},
\end{aligned}
$$

which implies that

$$
\lim _{n \rightarrow \infty} \int_{\sum_{1}^{n} C j+\gamma n}\left|k_{D}(t, z)-k_{D(n)}(t, z)\right|^{2} d s=0, \quad z \in D^{\prime} .
$$

From this we see that $\lim _{n \rightarrow \infty} k_{D(n)}(t, z)=k_{D}(t, z), z \in D^{\prime}$, almost everywhere on each $C_{\nu}$. Further we see that

$$
k_{D(n)}(z, w)=\frac{1}{2 \pi i} \int_{\sum_{1}^{n} C_{j}+r_{n}} \frac{k_{D(n)}(t, w)}{t-z} d t
$$

tends to 


$$
k_{D}(z, w)=\frac{1}{2 \pi i} \int_{C} \frac{k_{D}(t, w)}{t-z} d t
$$

uniformly in the wider sense. Here "in the wider sense" means originally that the convergence is uniform in any closed subdomain lying in $D^{\prime}-\bar{U}_{\varepsilon}(0)$. However it coincides with the ordinary one in this case. Similarly we see that $\rho_{D(n)}(z, w)$ tends to $\rho_{D}(z, w)$ uniformly in the wider sense. Thus this is also true for the function $l_{D(n)}(z, w)$, that is, $\lim _{n \rightarrow \infty} l_{D(n)}(z, w)=l_{D}(z, w)$.

It is well known that there exists uniquely an extremal function $F_{D(n)}(t, z)$ in $\mathfrak{B}_{D(n)}$ up to the rotation which gives $\sup _{\mathfrak{B}_{D(n)}}\left|g^{\prime}(z)\right|$ and this is connected with the kernels as follows:

$$
F_{D(n)}(t, z)=\frac{k_{D(n)}(t, z)}{l_{D(n)}(t, z)} .
$$

Therefore we see that

$$
F_{D}(t, z)=\frac{k_{D}(t, z)}{l_{D}(t, z)}
$$

exists and belongs to the class $\mathfrak{B}_{D}$ and $F_{D}(t, z)$ is an extremal function. Since we have for any $g \in \mathfrak{B}_{D}$

$$
\begin{aligned}
\left|g^{\prime}(z)\right| & =\left|2 \pi i \int_{C} l_{D}(t, z)^{2} g(t) d t\right| \leqq 2 \pi \int_{C}\left|l_{D}(t, z)\right|^{2} d s \\
& =2 \pi \int_{C} l_{D}(t, z)^{2} \frac{k_{D}(t, z)}{l_{D}(t, z)} d t=2 \pi \int_{C} l_{D}(t, z)^{2} F_{D}(t, z) d t \\
& =\frac{1}{i} F_{D}(z, z)=2 \pi k_{D}(z, z),
\end{aligned}
$$

the extremality and the uniqueness are obtained as usually. Of course we mean that the extremal function is unique up to the rotation. Now we have an affirmative answer to an exhaustion problem connected with the class $\mathfrak{B}$.

Next we enter into the second extremal problem, which is also well known in the case of finitely connected analytic domain. To seek for a function $f(z)$ such that $f^{\prime}(z)$ is regular except at $z=t$ around which the expansion

$$
-\frac{1}{2 \pi i(z-t)^{2}}+\text { regular terms of } z-t
$$

holds and $f^{\prime}(z)$ gives the minimum length

$$
\min \int_{C}\left|f^{\prime}(z)\right| d s \text {. }
$$

Here we only state the final result with no detail. The indefinite integral $f(z)$ of the function $f^{\prime}(z)=-2 \pi i l_{D}(z, t)^{2}$ is an extremal function of the problem and the minimum value is equal to $2 \pi k_{D}(t, t)$. Then we have

$$
\lim _{n \rightarrow \infty}-2 \pi i l_{D(n)}(z, t)^{2}=-2 \pi i l_{D}(z, t)^{2}
$$


uniformly in the wider sense in $D \times D$.

10. Here we shall study the boundary behavior of the kernels $k(z, t), \rho(z, t)$ and $l(z, t)$, in particular, their behavior when $t$ tends to the origin, which is the only one cluster point of the boundary components. In this $\S 10$ we settle a new assumption that

$$
\sum_{\nu=1}^{\infty} \frac{r_{\nu}\left(a_{\nu}{ }^{2}+r_{\nu}{ }^{2}\right)}{\left(a_{\nu}{ }^{2}-r_{\nu}^{2}\right)^{3}}<\infty
$$

holds. From (C) we have

$$
\sum_{\nu=1}^{\infty} \frac{r_{\nu}}{a_{\nu}^{2}-r_{\nu}^{2}}<\infty
$$

which implies that $1 / z \in L^{2}$.

In the first place we prove that

$$
\frac{1}{2 \pi i} \int_{C} \frac{d z}{z(z-t)}= \begin{cases}0, & t \in D, \\ -1 / t, & t \in B .\end{cases}
$$

For the left hand side integral $I$ we have

$$
\begin{aligned}
|I| & \leqq \frac{1}{2 \pi} \int_{C} \frac{d s}{|z||z-t|} \leqq \frac{1}{2 \pi}\left(\int_{C} \frac{d s}{|z|^{2}}\right)^{1 / 2}\left(\int_{C} \frac{d s}{|z-t|^{2}}\right)^{1 / 2} \\
& =\frac{1}{2 \pi}\left(\sum_{\jmath=1}^{\infty} \frac{2 \pi r_{\jmath}}{\left|a_{\jmath}\right|^{2}-r_{\jmath}^{2}}\right)^{1 / 2}\left(\sum_{\jmath=1}^{\infty} \frac{2 \pi r_{\jmath}}{\left|a_{\jmath}-t\right|^{2}-r_{\jmath}^{2}}\right)^{1 / 2}, \quad t \in D .
\end{aligned}
$$

If $t$ lies in a closed subdomain of $D$, then

$$
\sum_{j=1}^{\infty} \frac{r_{j}}{\left|a_{j}-t\right|^{2}-r_{j}{ }^{2}} \leqq N<\infty
$$

holds and hence the integral $I$ exists absolutely and uniformly in the wider sense in $D$. This shows that

$$
I=\lim _{n \rightarrow \infty} \sum_{j=1}^{n} \int_{C_{j}} \frac{d z}{z(z-t)}=0 .
$$

If $t \in B$, then the integral $I$ also exists absolutely and uniformly in the wider sense in $B_{\nu}$. This shows that

$$
I=\lim _{n \rightarrow \infty} \sum_{j=1}^{n} \int_{C j} \frac{d z}{z(z-t)}=-\frac{2 \pi i}{t} .
$$

We decompose $1 / 2 \pi i z$ in such a manner that

$$
\frac{1}{2 \pi i z}=\rho_{1}(z)+\overline{k_{1}(z) z^{\prime}}, \quad z \in C, \quad \rho_{1}(z) \in \Gamma^{2}
$$

and

$$
\min _{\mu \in \Gamma^{2}}\left\|\frac{1}{2 \pi i z}-\mu(z)\right\|^{2}=\left\|\frac{1}{2 \pi i z}-\rho_{1}(z)\right\|^{2}=\left\|k_{1}(z)\right\|^{2}
$$


Then as in $\S \S 5$ and 8 we have $k_{1}(z) \in \Lambda^{2} \cap \Phi, \rho_{1}(z) \in \Gamma^{2}{ }_{n} \Phi$ and the analyticity of $k_{1}(z), \rho_{1}(z)$ and $l_{1}(z)$ on each $C_{n}$, where $l_{1}(z)=1 / 2 \pi i z-\rho_{1}(z)$. And further we have $l_{1}(z)=\overline{k_{1}(z) z^{\prime}}$ on each $C_{n}$. By the orthogonality $l(z, t) \perp \Gamma^{2}$ and $l_{1}(z)$ $\perp \Gamma^{2}$, we have

$$
\overline{\frac{1}{2 \pi i} \int_{C} \frac{\overline{\rho(z, t)}}{z} d s}=\int_{C} \overline{\rho_{1}(z)} \rho(z, t) d s=\frac{1}{2 \pi i} \int_{C} \frac{\overline{\rho_{1}(z)}}{z-t} d s,
$$

and hence we have

$$
\begin{aligned}
k_{1}(t) & =\frac{1}{2 \pi i} \int_{C} \frac{k_{1}(z)}{z-t} d z=\frac{1}{2 \pi i} \int_{C} \frac{\overline{l_{1}(z)}}{z-t} d s \\
& =\frac{1}{4 \pi^{2}} \int_{C} \frac{d s}{\bar{z}(z-t)}-\frac{1}{2 \pi i} \int_{C} \frac{\overline{\rho_{1}(z)}}{z-t} d s \\
& =\frac{1}{4 \pi^{2}} \int_{C} \frac{d s}{\bar{z}(z-t)}+\frac{1}{2 \pi i} \int_{C} \frac{\rho(z, t)}{\bar{z}} d s .
\end{aligned}
$$

Therefore we see that

$$
k(t, w)-k_{1}(t)=\bar{w}\left[\frac{1}{4 \pi^{2}} \int_{C} \frac{d s}{(z-t) \bar{z}(\bar{z}-\bar{w})}+\frac{1}{2 \pi i} \int_{C} \rho(z, t) \frac{d s}{\bar{z}(\bar{z}-\bar{w})}\right] .
$$

Let $m$ be the minimum distance $\min _{z \in C}|z-t|$, then there holds

$$
\left|\int_{C} \frac{d s}{(z-t) \bar{z}(\bar{z}-\bar{w})}\right| \leqq \frac{1}{m} \int_{C} \frac{d s}{|z||z-w|}<\infty
$$

for any $w$ belonging to the angular domain $\Theta: \pi / 2+\varepsilon \leqq \arg w \leqq 3 \pi / 2-\varepsilon$ for an $\varepsilon>0$. And further we have

$$
\left|\int_{C} \rho(z, t) \frac{d s}{\bar{z}(\bar{z}-\bar{w})}\right|^{2} \leqq \int_{C}|\rho(z, t)|^{2} d s \int_{C} \frac{d s}{|z-w|^{2}|z|^{2}} \leqq M^{2} \int_{C} \frac{d s}{|z|^{4}}
$$

for any $w$ lying in the angular domain $\Theta$. On the other hand we have easily

$$
\int_{C} \frac{d s}{|z|^{4}}=2 \pi \sum_{\nu=1}^{\infty} \frac{r_{\nu}\left(a_{\nu}^{2}+r_{\nu}^{2}\right)}{\left(a_{\nu}{ }^{2}-r_{\nu}^{2}\right)^{3}}<\infty .
$$

Therefore under the condition (C) the integral

$$
\int_{C} \rho(z, t) \frac{d s}{\bar{z}(\bar{z}-\bar{w})}
$$

exists absolutely and uniformly in $\Theta$. Therefore $\lim _{w \rightarrow 0} k(t, w)=k_{1}(t)$ holds, where the limit is taken over the point-sequence lying in the angular domain $\Theta$, and moreover we see that the differentiability

$$
\begin{aligned}
\left.\frac{\partial}{\partial \bar{w}} k(t, w)\right|_{w=0} & =\lim _{w \rightarrow 0} \frac{k(t, w)-k_{1}(t)}{\bar{w}} \\
& =\frac{1}{4 \pi^{2}} \int_{C} \frac{d s}{(z-t) \bar{z}^{2}}+\frac{1}{2 \pi i} \int_{C} \frac{\rho(z, t)}{\bar{z}^{2}} d s \\
& =\frac{-1}{2 \pi i} \int_{C} \frac{1}{\bar{z}^{2}} l(z, t) d s=\frac{-1}{2 \pi i} \int_{C} \frac{1}{\bar{z}^{2}} \overline{k(z, t)} d z
\end{aligned}
$$


holds for any limiting process in $\Theta$. And again by the orthogonality $l(z, t)$ $\perp k_{1}(z)$, we have

$$
\rho_{1}(t)=\frac{1}{2 \pi i} \int_{C} \frac{\rho_{1}(z)}{z-t} d z=-\frac{1}{2 \pi i} \int_{C} \frac{\rho(z, t)}{z} d z,
$$

from which we can see that $\lim _{w \rightarrow 0} \rho(t, w)=\rho_{1}(t), w \in \Theta$ and

$$
\lim _{w \rightarrow 0} \frac{\rho(t, w)-\rho_{1}(t)}{w}=-\frac{1}{2 \pi i} \int_{C} \frac{\rho(z, t)}{z^{2}} d z, \quad w \in \Theta
$$

under the condition (C). This implies that $\lim _{w \rightarrow 0} l(t, w)=l_{1}(t), w \in \Theta$ and

$$
\begin{aligned}
\lim _{w \rightarrow 0} \frac{l(t, w)-l_{1}(t)}{w} & =\frac{1}{2 \pi i t^{2}}-\lim _{w \rightarrow 0} \frac{\rho(t, w)-\rho_{1}(t)}{w} \\
& =\frac{1}{2 \pi i t^{2}}+\frac{1}{2 \pi i} \int_{C} \frac{\rho(z, t)}{z^{2}} d z, \quad w \in \Theta .
\end{aligned}
$$

Since there holds an identity

$$
\int_{C} \frac{d z}{z^{2}(z-t)}=0, \quad t \in D
$$

by the condition (C) and by the residue theorem, we have

$$
\left.\frac{\partial}{\partial w} l(t, w)\right|_{w=0}=\frac{1}{2 \pi i t^{2}}-\frac{1}{2 \pi i} \int_{C} \frac{\overline{k(z, t)}}{z^{2}} d s .
$$

Let $\varphi$ be any function of the class $\Phi$. Then we have

and

$$
\left(\varphi(z), k_{1}(z)\right)=\int_{C} l_{1}(z) \varphi(z) d z=\frac{1}{2 \pi i} \int_{C} \frac{\varphi(z)}{z} d z
$$

$$
\left(\varphi(z), k(z, w)-k_{1}(z)\right)=\frac{w}{2 \pi i} \int_{C} \varphi(z) \frac{d z}{z(z-w)},
$$

whence follows that

and further that

$$
\left(\varphi(z), k_{1}(z)\right)=\lim _{w \rightarrow 0} \varphi(w), \quad w \in \Theta
$$

$$
\left\|k(z, w)-k_{1}(z)\right\|^{2} \leqq \frac{|w|}{2 \pi}\left\|k(z, w)-k_{1}(z)\right\|\left(\int_{C} \frac{d s}{|z|^{2}|z-w|^{2}}\right)^{1 / 2}
$$

This shows that the strong convergence of $k(z, w)$ to $k_{1}(z)$ :

$$
\lim _{w \rightarrow 0}\left\|k(z, w)-k_{1}(z)\right\|^{2}=0, \quad w \in \Theta
$$

holds, from which we see that $\lim _{w \rightarrow 0} k_{1}(w)=\left\|k_{1}(z)\right\|^{2}, w \in \Theta$ and $\lim _{w \rightarrow 0} k(w, w)$ $=\left\|k_{1}(z)\right\|^{2}, w \in \Theta$.

Suppose further that there remains valid

$$
\int_{C} \frac{d s}{|z|^{6}}<\infty
$$


Then there holds

$$
\int_{C}\left(\frac{k_{1}(z)}{z(z-w)}-\frac{k_{1}(z)}{z^{2}}\right) d z=w \int_{C} \frac{k_{1}(z)}{z^{2}(z-w)} d z \rightarrow 0
$$

as $w$ tends to the origin in $\Theta$. Moreover it is evident that

$$
\left|\int_{C} \frac{k(z, w)-k_{1}(z)}{z(z-w)} d s\right|^{2} \leqq\left\|k(z, w)-k_{1}(z)\right\|^{2} \int_{C} \frac{d s}{|z|^{2}|z-w|^{2}}=O\left(|w|^{2}\right)
$$

as $w$ tends to the origin in $\Theta$. These show that

$$
\lim _{w \rightarrow 0} \frac{k(w, w)-k_{1}(w)}{w^{2}}=\frac{1}{2 \pi i} \int_{C} \frac{k_{1}(z)}{z^{3}} d z, \quad w \in \Theta .
$$

If $1 / z^{n}$ belongs to the class $L^{2}$ for any positive integer $n$, then we can proceed these processes ad infinitum. The example 2 in $\S 7$ satisfies this condition.

11. Let us decompose $1 / 2 \pi i z$ in such a manner that

$$
\frac{1}{2 \pi i z}=P_{1}(z)+\overline{K_{1}(z) z^{\prime}}, \quad P_{1}, K_{1} \in A^{2}
$$

and that

$$
\left\|\frac{1}{2 \pi i z}-P_{1}(z)\right\|^{2}=\min _{\mu \in \Lambda^{2}}\left\|\frac{1}{2 \pi i z}-\mu\right\|^{2},
$$

then we have $P_{1}, K_{1} \in \Phi$ and both are analytic on each $C_{n}$ with the boundary relation $L_{1}(z)=\overline{K_{1}(z) z^{\prime}}$ where we put $L_{1}(z)=1 / 2 \pi i z-P_{1}(z)$. And further we have the orthogonality $L_{1}(z) \perp \Lambda^{2}$. Therefore we have

$$
\begin{aligned}
\frac{1}{2 \pi i} \int_{C} \overline{k(t, w)} \frac{d s}{t} & =\int_{C} \overline{k(t, w)} P_{1}(t) d s+\overline{\int_{C} k(t, w) K_{1}(t) d t} \\
& =P_{1}(w)+\overline{\int_{C} k(t, w) \overline{L_{1}(t)} d s}=P_{1}(w) .
\end{aligned}
$$

If we assume that

$$
\frac{1}{2 \pi i} \int_{C} \overline{k(t, w)} \frac{d s}{t}=\frac{1}{2 \pi i w}
$$

remains valid, then $P_{1}(w)=1 / 2 \pi i w$. However we have

$$
\int_{C} \frac{P_{1}(w)}{w-t} d w=0, \quad t \in B
$$

and

$$
\frac{1}{2 \pi i} \int_{C} \frac{d w}{w(w-t)}=-\frac{1}{t}, \quad t \in B
$$

which is a contradiction. Thus we have a remarkable fact that there is a function regular in $D$ and belonging to the class $L^{2}$, for which the reproducing 
property does not hold. The class $\Phi$ plays a central role for the reproducing property.

Our results are of very special nature, since the boundary of the domain consists of the circles and their centres lie on the real axis and the order of clustering is very weak in our case. We have introduced two notions, that is, "of finite type" and "of infinite island type", which rshow the orders of clusteing of the circles, respectively.

There arise several unsolved problems, which are listed below.

(a) Can we extend the condition of finite type to a more general domain than our cases presented here? It should be hoped that the condition to be established is of purely metrical nature.

(b) Under what condition is the extremal function $F(t, z)$ unique? We have established this under the condition of infinite island type and of finite type.

(c) Under what condition is the extremal function $F(t, z)$ of type $B l_{1}$ in the sense of Heins [4]? It is our final conjecture that the extremal function $F(t, z)$ is of type $B l_{1}$ in a case of infinite island type and simultaneously of finite type.

\section{REFERENCES}

[1] Ahlfors, L., AND A. Beurling, Conformal invariants and function-theoretic null-sets. Acta Math. 83 (1950), 101-129.

[2] Garabedian, P. R., Schwarz's lemma and the Szegö kernel function. Trans. Amer. Math. Soc. 67 (1949), 1-35.

[3] Garabedian, P. R., AND M. Schiffer, On existence theorems of potential theory and conformal mapping. Ann. of Math. 52 (1950), 164-187.

[4] HeINs, M., On the Lindelöf principle. Ann. of Math. 55 (1955), 296-317.

[5] Nehari, Z., On bounded analytic functions. Proc. Amer. Math. Soc. 1 (1950), 268-275.

[6] SCHIFFER, M., Various types of orthogonalization. Duke Math. Journ. 17 (1950), 329-366.

[7] SingH, V., An integral equation associated with the Szegö kernel function. Proc. London Math. Soc. (3) 10 (1960), 376-393.

Department of Mathematics, Tokyo Institute of TeChNology. 